\title{
Repurposing Molecular Imaging and Sensing for Cancer Image-Guided Surgery
}

\author{
Suman B. Mondal*1, Christine M. O’Brien*1, Kevin Bishop ${ }^{1}$, Ryan C. Fields ${ }^{2}$, Julie A. Margenthaler ${ }^{2}$, \\ and Samuel Achilefu ${ }^{1,3,4}$ \\ ${ }^{1}$ Department of Radiology, Washington University, St. Louis, Missouri; ${ }^{2}$ Department of Surgery and Siteman Cancer Center, \\ Washington University School of Medicine, St. Louis, Missouri; ${ }^{3}$ Department of Biomedical Engineering, Washington University, \\ St. Louis, Missouri; and ${ }^{4}$ Department of Biochemistry and Molecular Biophysics, Washington University, St. Louis, Missouri
}

\begin{abstract}
Gone are the days when medical imaging was used primarily to visualize anatomic structures. The emergence of molecular imaging (MI), championed by radiolabeled ${ }^{18} \mathrm{~F}-\mathrm{FDG} \mathrm{PET}$, has expanded the information content derived from imaging to include pathophysiologic and molecular processes. Cancer imaging, in particular, has leveraged advances in $\mathrm{Ml}$ agents and technology to improve the accuracy of tumor detection, interrogate tumor heterogeneity, monitor treatment response, focus surgical resection, and enable image-guided biopsy. Surgeons are actively latching on to the incredible opportunities provided by medical imaging for preoperative planning, intraoperative guidance, and postoperative monitoring. From label-free techniques to enabling cancer-selective imaging agents, imageguided surgery provides surgical oncologists and interventional radiologists both macroscopic and microscopic views of cancer in the operating room. This review highlights the current state of Ml and sensing approaches available for surgical guidance. Salient features of nuclear, optical, and multimodal approaches will be discussed, including their strengths, limitations, and clinical applications. To address the increasing complexity and diversity of methods available today, this review provides a framework to identify a contrast mechanism, suitable modality, and device. Emerging low-cost, portable, and user-friendly imaging systems make the case for adopting some of these technologies as the global standard of care in surgical practice.
\end{abstract}

Key Words: research methods; Cerenkov luminescence; fluorescence imaging; molecular image-guided surgery; multimodal imaging; nuclear imaging

J Nucl Med 2020; 61:1113-1122

DOI: 10.2967/jnumed.118.220426

\section{$\mathbf{M}$} edical imaging is central to major advances witnessed in modern medicine, allowing visualization of the entire human body. With the advent of molecular imaging (MI), it is now possible to see beyond what is accessible to the naked eye, including metabolic and molecular processes. A combination of high-resolution anatomic imaging and high-sensitivity MI allows accurate localization and

Received Dec. 29, 2019; revision accepted Mar. 5, 2020.

For correspondence or reprints contact: Samuel Achilefu, Department of Radiology, Washington University, CB 8225, Room 1150, East Building, 4525 Scott Ave., St. Louis, MO 63110.

E-mail: achilefu@wustl.edu

${ }^{*}$ Contributed equally to this work.

COPYRIGHT (c) 2020 by the Society of Nuclear Medicine and Molecular Imaging. in-depth interrogation of biomarker status. A notable achievement of imaging advances is the deescalation in the need for exploratory surgery, an invasive process that is used to determine treatment planning. Until recently, there remained a dearth of information regarding the direct application of MI in the operating room (OR). Despite the availability of exquisite preoperative images, surgeons relied on visual and tactile tissue assessment to inform surgical decisions, partly because of tissue deformation that causes a significant mismatch between the visible tissue of interest and the noninvasive preoperative image. Improved adaptation of current imaging technologies in the operative room has led to a surge in imageguided surgery, particularly in surgical oncology. One of the first such integrated radiology-surgery systems, the Advanced Multimodality Image Guided Operating suite, was developed at Brigham and Women's Hospital in Boston (1). The platform allows collaborative medicine in which radiologists, surgeons, and oncologists can work as a team before, during, and after surgical interventions. This suite uses MRI and PET/CT systems as the imaging units. With recent efforts to miniaturize imaging systems and minimize the footprint in the OR, new label-free and molecular contrast-enhanced technologies for real-time intraoperative image-guided surgery have emerged (2-5). Whereas label-free approaches harness the intrinsic properties of tissue to delineate cancer from healthy tissue, contrast agentenhanced techniques amplify imaging signal in the tumor for identifying cancer boundaries.

Irrespective of the modality used, methods for intraoperative image guidance require a sensor device to capture the imaging signal. Spontaneously generated signals such as those from radionuclides do not require the additional input to produce signals that is required for most optical (chromophore excitation) and MR (radiofrequency perturbation) imaging methods. Although spontaneous signals simplify the hardware design and improve signal quantification, the lack of control over when to generate the signal limits the depth of information that can be derived from these agents for accurate delineation of tumor boundaries. Although functional MRI and other imaging systems are increasingly explored for intraoperative image guidance, we will limit this review to MI applications, focusing on optical and nuclear imaging modalities, which are widely used clinically for molecular image-guided surgery (MIGS) (Supplemental Table 1; supplemental materials are available at http://jnm.snmjournals.org). In the context of this review, we will discuss MI strategies that cover the use of exogenous imaging agents to interrogate specific cancer biomarkers and the sensing of endogenous or exogenous molecules that report the location or status of tumors for MIGS. Nuclear 
imaging enables quantitative deep-tissue imaging using radiotracers $(3,4)$ and is adaptable to rapid assessment of shallow tissue in the OR. Optical imaging, however, does not interrogate deep tissue with useful spatial resolution but allows high-resolution real-time imaging at shallow depths using nonionizing radiation (2-4). Although each of these modalities can be used independently, their complementary features have led to the development of multimodal approaches that combine their unique strengths (6). We will highlight recent advances in MI modalities, contrast agents, and imaging devices that have been clinically evaluated for surgical guidance in human cancer patients, and we will conclude with a framework to identify the most suitable intraoperative imaging approach to accomplish specific endpoints.

\section{CONTRAST}

Preoperative MI methods use multiple factors to uncover the presence of tumors, determine the stage of cancer, monitor treatment response, and identify residual tumors. Detecting large tumors using standard imaging methods is straightforward. However, recent advances in cancer biology have uncovered new molecular processes and imageable biomarkers that facilitate the interrogation of small tumors at the molecular level. Aided by minimally invasive biopsy and histologic validation, the goal of MI is not only to detect tumors but also to delineate malignant from benign lesions. These advances reduce the need for exploratory surgery and may improve the ability to perform more focused resections that remove less normal tissue. Instead, the task of surgical oncology is to remove tumors in an oncologically sound manner (i.e., removing the tumor and regional lymph nodes, when appropriate, to achieve a microscopically margin-negative resection). Key attributes of MI methods for surgery include the ability to display tumors rapidly, identify the tumor margins, detect microscopic lesions, be reproducible, and assess the surgical cavity for the presence of residual tumors. How well these needs are met depends on the source of contrast between cancer and the surrounding tissue. Thus, a significant amount of effort in MI is focused on identifying the best contrast mechanism to achieve these goals. Regardless of the source of contrast, the detected signal should improve the accuracy of cancer detection and resection without casting undue burden on the normal surgical procedure. The sources of imaging contrast can be either endogenous or exogenous to the patient (Fig. 1).

\section{Endogenous Contrast}

Endogenous contrast consists of biomolecules that are differentially expressed in cancer compared with the surrounding tissue $(3,4)$ (Supplemental Table 2). This mechanism is not available

\section{NOTEWORTHY}

- MI-guided surgery enhances real-time cancer detection and characterization.

- A variety of contrast mechanisms highlights unique features of cancer.

- Diverse imaging systems and contrast agents provide flexibility in the choices available to clinicians.

- Standardization of imaging systems and reporting strategy will facilitate clinical adoption.

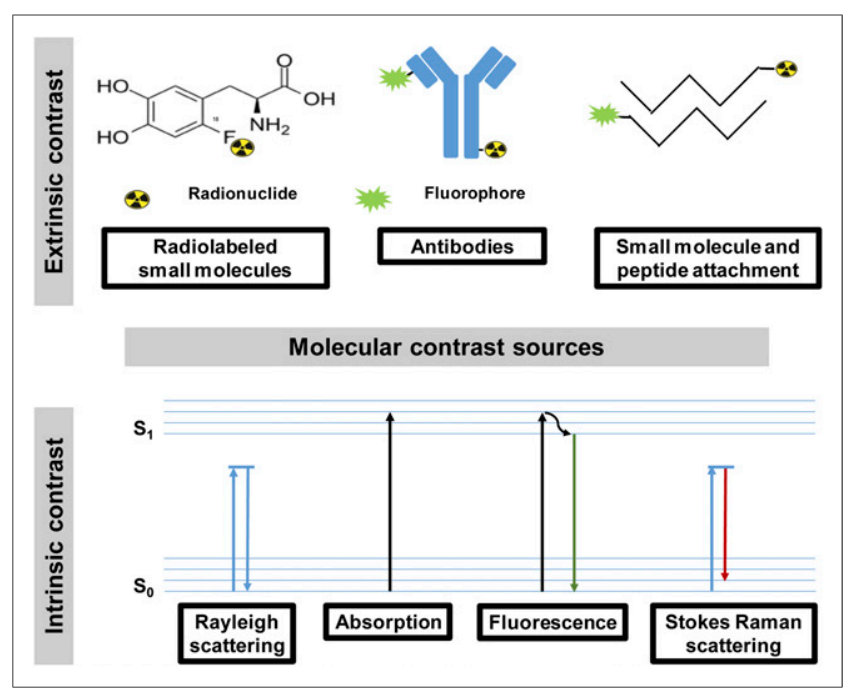

FIGURE 1. Sources of extrinsic and intrinsic contrast used in molecular image-guided surgery. $\mathrm{s}_{1}=$ first excited singlet state; $\mathrm{s}_{0}=$ singlet ground state.

to nuclear imaging because the method relies on exogenously administered radioactivity for contrast. In comparison, optical and hybrid technologies can harness intrinsic signals to generate contrast and are the major beneficiaries of endogenous contrast (710). Many proteins play a major role in cancer survival, some of which can absorb, scatter, or emit light of specific wavelengths. This process can produce a variety of detectable imaging signals. Excitation of tissue with light of a specific wavelength range yields red-shifted autofluorescence for imaging tissue at shallow depths. Spectroscopic methods have been developed to detect specific features of some endogenous molecules. For example, Raman spectroscopy probes molecular bond vibrations by measuring inelastically scattered light, which reports molecular signatures of tissue. Similarly, diffuse reflectance spectroscopy leverages the ability of some biomolecules to scatter and absorb light in a wavelength-dependent manner to determine tissue composition and identify cancer. Another exciting method, optical coherence tomography, uses interference patterns generated by light scatter and absorption as it passes through tissue to identify tissue microstructure. Classic optical coherence tomography is not directly sensitive to molecular information in tissue, and thus several innovative approaches have been introduced to pair the high morphologic resolution with functional content (11). Although it has not gained acceptance for MIGS, it is only a matter of time before this powerful technique makes an impact in human surgery.

Some optical hybrid techniques such as photoacoustic imaging use molecular absorption by biologic chromophores to induce localized pressure waves created by thermoelastic expansion and detected by ultrasound probes. These sources of contrast provide diverse signaling mechanisms to improve the accuracy of cancer surgery. We will explore their applications in the surgical environment in the sections dedicated to each of these MI modalities.

\section{Exogenous Contrast}

Exogenous contrast agents are administered into the patient, typically via intravenous or intraperitoneal routes, and accumulate in tumors for a few minutes or several days, depending on the intended application. The tumor uptake varies from a passive 
accumulation and the sensing of specific enzyme activity to the selective binding of upregulated proteins on cancer cells (Supplemental Table 2) (1,2,12). Lymph node-seeking agents such as radiocolloids are used to highlight sentinel lymph nodes (SLNs) as part of cancer staging (13). The multifunctionality of colloidal and nanoparticles is attractive for both active and passive targeting of tumors for image-guided surgery. The excitement about the targeting of imageable cancer biomarkers has stimulated interest in the development of many radiopharmaceuticals and fluorescent dye conjugates of tumor-targeting small molecules, peptides, and antibodies $(12,14)$. In general, contrast agents that have short half-lives (minutes) may not be suitable for MIGS for logistic reasons, including time from the imaging suite to the OR. An ideal agent for surgical guidance is expected to accumulate rapidly in tumors, have high tumor-to-background contrast, and be retained in this state for at least $4 \mathrm{~h}$. The ability to optimize the pharmacokinetics of small- and medium-sized molecules such as peptides (15-17) and antibody fragments (18) makes them attractive for this purpose. Although some researchers have argued against the use of antibodies for imaging applications because of the long circulation time (up to $7 \mathrm{~d}$ ), recent studies have leveraged the exceptional high tumor-associated receptors to identify small lesions (19). Moreover, with improved dosing regimens, these molecules can provide adequate tumor-to-background contrast within a few hours after injection. To synergize the strengths of different imaging modalities, multimodal contrast agents are beginning to find their way into the $\operatorname{OR}(1,20)$. This trend bodes well for oncologic surgery, as the combination of deep-tissue profiling to assess the extent of tumor infiltration and highly sensitive detection schemes for superficial lesions will enable rapid identification of cancer boundaries. In the future, we expect the integration of endogenous with exogenous contrast mechanisms to further improve the accuracy of cancer surgery and real-time assessment of surgical margins.

\section{DEVICES}

The current standard of care requires surgeons to rely on visual inspection, palpation, and tactile evaluation to identify cancer. Image guidance would accelerate tumor identification and provide information about possible infiltration, thereby enhancing the ability to achieve a microscopically margin-negative (R0) resection with high reproducibility, ease of use, and enhanced throughput. However, the evolving landscape of surgical precision and rigor requires accurate and efficient removal of tumors with negative margins. These cells are typically microscopic and invisible to the naked eye. In some tumor types, intraoperative assessment of surgical margins further drives high contrast and quantitative invasive techniques to improve surgical outcomes. Sensing this opportunity, many research groups and companies are developing small-footprint imaging systems for guiding oncologic surgery. Whereas $\gamma$-cameras are optimized for detecting radioactivity in the OR, detectors used in optical and ultrasound imaging are diverse, differing in the sensor and the wavelength ranges captured to the system configurations (Fig. 2), which determine MIGS sensitivity, user experience, and clinical adoption potential.

\section{Standalone}

Standalone configurations are the most common design of MIGS systems (Supplemental Table 3). They typically use an articulating arm supporting illumination (in the case of optical) and detection hardware, with image-processing and display

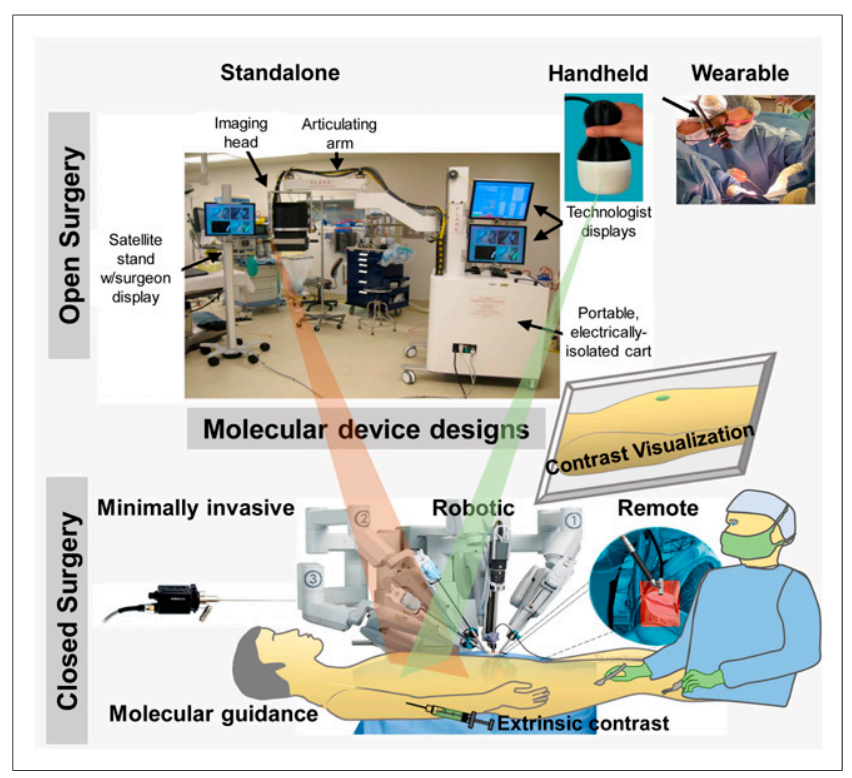

FIGURE 2. Design configurations for molecular image-guided surgical devices, including standalone (22), handheld (25), wearable (arrow) (28), minimally invasive (30), and robotic (36).

hardware integrated in a wheeled tower (19,21-23). Their fixed arm position simplifies image alignment but provides only a topdown field of view that requires surgeons to mentally correlate the displayed molecular information to the anatomic region of interest (ROI). Remote image display also forces surgeons to transiently look away from the surgical bed, a subtle distraction that cumulatively prolongs surgery. To address these issues, systems that project molecular information directly on the anatomic ROI have been developed (24). The simple design and image display of this platform is attractive for MIGS, but the large size disrupts surgical workflow in the current form. Future miniaturization of the system will overcome this impediment and reduce cost.

\section{Handheld}

Handheld devices use a miniature, portable imaging-hardware configuration that performs image processing and display on a wheeled tower. Their design allows easier surgical workflow integration and access for hard-to-reach anatomic ROIs than do fixed standalone systems. A variety of these systems is currently commercially available for MIGS (Supplemental Table 3). These devices are generally less expensive than the standalone systems and provide an intuitive user experience $(21,25-27)$. However, the system does not support hands-free surgery, implying that the operation will stop when the system is used by the operating surgeon. Furthermore, when held by a surgical team member or fixed to an articulating arm, the image and the surgeon's field of view are mismatched. Familiarity with the system improves the ease of use and adoption.

\section{Wearable}

Wearable devices use miniaturized imaging detectors, powerful processors, and fast image-processing to display real-time molecular information to surgeons via head-mounted displays. Direct projection of the molecular information to the surgeon's field of view enables seamless integration to the intraoperative surgical workflow $(28,29)$. Head-mounted displays are increasingly used 
for medical image visualization in mixed-reality applications, and their small size, low cost, and intuitive user experience make them very amenable for wide clinical adoption (Supplemental Table 3). When the illumination module is mounted on the head-mounted display, the devices provide complete hands-free surgery, allowing the operating surgeon to have full control of the system during MIGS. Real-time display of the images on remote monitors enhances collaboration with a surgical team. Key considerations to enhance the user experience and wide adoption for head-mounted displays include weight, size, and ergonomics.

\section{Minimally Invasive}

The rise and expansion of minimally invasive surgery (robotic and laparoscopic) have decreased the invasiveness and morbidity of surgery. The adaptation of imaging devices to minimally invasive platforms has been rapid and necessary to enhance imaging techniques in these procedures. Minimally invasive devices combine MI hardware with endoscopes to visualize and remove suspected lesions inside the body (30-33). Traditional endoscopy uses white light to identify the location of tumors, but the integration of illumination modules and appropriate filters into existing or new endoscopes transforms the procedure into a theranostic platform for tumor detection, assessment, and removal. Endoscopic surgeries are increasingly being adapted for MIGS (Supplemental Table 3). Current devices process and display images on a wheeled tower with an integrated or wall monitor. This remote analysis can distort data fidelity and decrease the detection sensitivity due to loss of signal. To overcome these challenges, newer devices incorporate sensors at the front end of the endoscope. The need to directly visualize anatomic structures during endoscopy has led to the development of computational depth perception approaches (34). However, coregistration of the image field of view with patient anatomy using preoperative structural images is complicated by tissue deformation, and obtaining intraoperative structural images is challenging because of hardware complexity. To address these challenges, computational reconstruction of local 3-dimensional tissue structure has been developed for CT mapping (35). With the increasing adoption of minimally invasive surgery, there will be an increasing focus on this configuration for MIGS.

\section{Robotic and Remote}

Robotic systems use minimally invasive devices controlled by a surgeon through a robotic interface that controls multiple surgical ports and uses image data to guide surgical decisions (Supplemental Table 3). Such systems lend themselves naturally to remote guidance, making them popular for integration with other modalities $(6,36,37)$. These devices are revolutionizing surgical care by improving the accuracy of tumor resection and extending the working lifespan of surgeons. They are also useful in lowresource settings by enabling remote surgical guidance. However, high cost and lengthy training time remain major limitations to wide clinical adoption.

\section{NUCLEAR IMAGING}

Palpable cancer is easy to detect in the OR, but some cancer types often present as nonpalpable lesions. Radioguided surgery was developed to overcome this problem (Fig. 3). Not only is this technique capable of aiding tumor localization in the OR, but it also affords the opportunity to detect occult disease and SLNs using $\gamma$-probes or cameras (Supplemental Table 4) (38). Radiocolloid

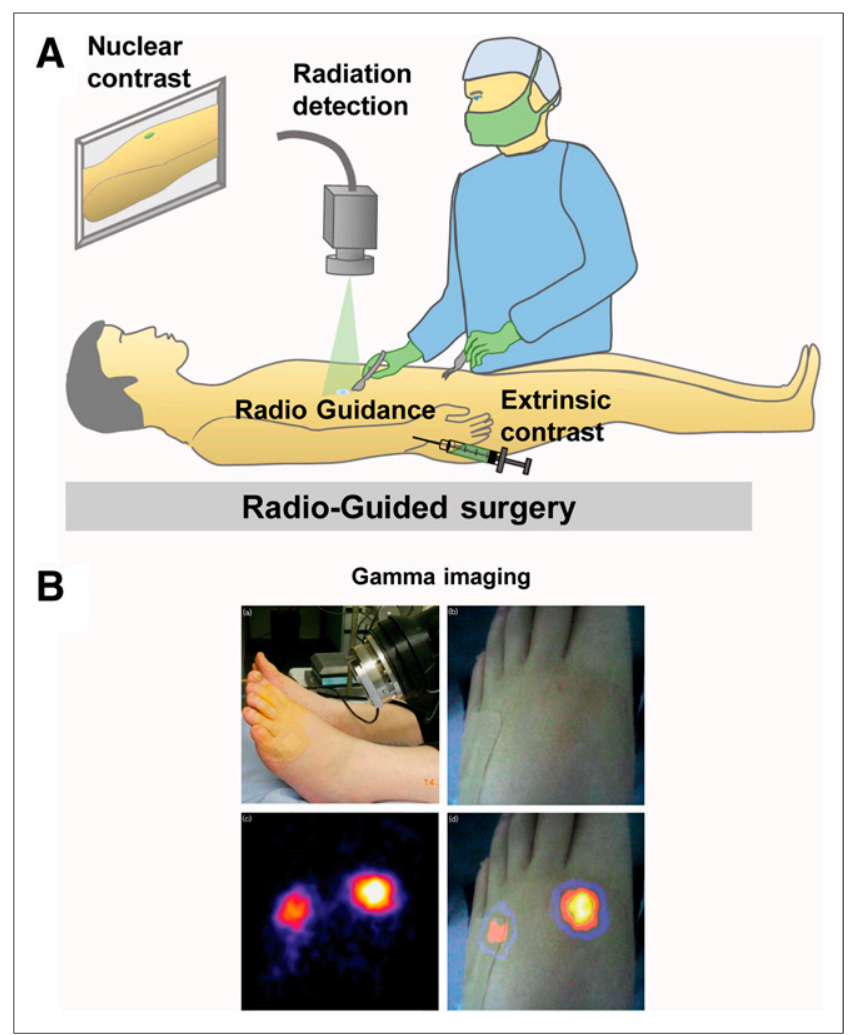

FIGURE 3. Radioguided surgery: schematic representation (A) and clinical example of y-imaging-guided surgery (20) (B).

lymphoscintigraphy is the precursor to $\gamma$-imaging radioguided surgery, serving as an alternative to guide wire-localized breast surgery. In this technique, handheld scintillation probes provide an audible output when detecting low- or medium-energy (140-250 keV) $\gamma$-rays from SPECT radiotracers (39). Radioguided-surgery occult lesion localization $(40)$ and SLN biopsy $(41,42)$ using handheld $\gamma$-probes are now the standard of care for breast cancer and melanoma surgery, with increasing adoption by other surgical specialties. Recently, handheld PET probes capable of detecting high-energy (511 keV) $\gamma$-rays have been evaluated for tumor detection (43). Lack of visual feedback in these probes led to the development of portable $\gamma$-cameras that have allowed the identification of additional SLNs, including metastatic SLNs that were missed by the conventional audio output $\gamma$-probe (44). Small-field-of-view hybrid $\gamma$-optical cameras (25) now allow dual-channel imaging and superimposed high-resolution nuclear-fluorescence image visualization (20). Freehand SPECT has also been used to guide SLN biopsy during breast (45) and oral (46) cancer surgeries. When used in conjunction with cancer-targeting radiopharmaceuticals (14), radioguided surgery is an excellent method to rapidly identify cancer, guide tumor resection, and survey the cavity after bulk tumor removal to minimize residual disease. A considerable limitation of this approach is the regulatory hurdles to use and maintain radiopharmaceuticals, confining this technique to specialized surgical centers.

\section{OPTICAL IMAGING}

Optical imaging uses nonionizing radiation to illuminate endogenous or exogenous sources of contrast and enables real-time, high-resolution 
MIGS (2-4). Many sources and mechanisms of contrast are available for optical imaging methods, leading to the development of different techniques for this modality (Fig. 4). Below, we have highlighted the methods that are widely used in MIGS while acknowledging that other newer methods are equally valuable.

\section{Fluorescence}

Autofluorescence. Autofluorescence imaging takes advantage of naturally occurring fluorophores in the body, whose differential expression in healthy and cancerous tissue provides contrast for surgical guidance (Supplemental Table 5). Unlike methods that rely on exogenous contrast agents, the path to clinical use of autofluorescence-guided surgery is simpler, with instrument optimization and data analysis methods as the primary optimization task. Autofluorescence has seen strong application in detecting head and neck cancers, especially in the oral cavity, by visualizing loss of signal in cancerous tissue due to altered metabolism (7). This principle enabled the use of a simple handheld autofluorescence imaging device to accurately detect oral

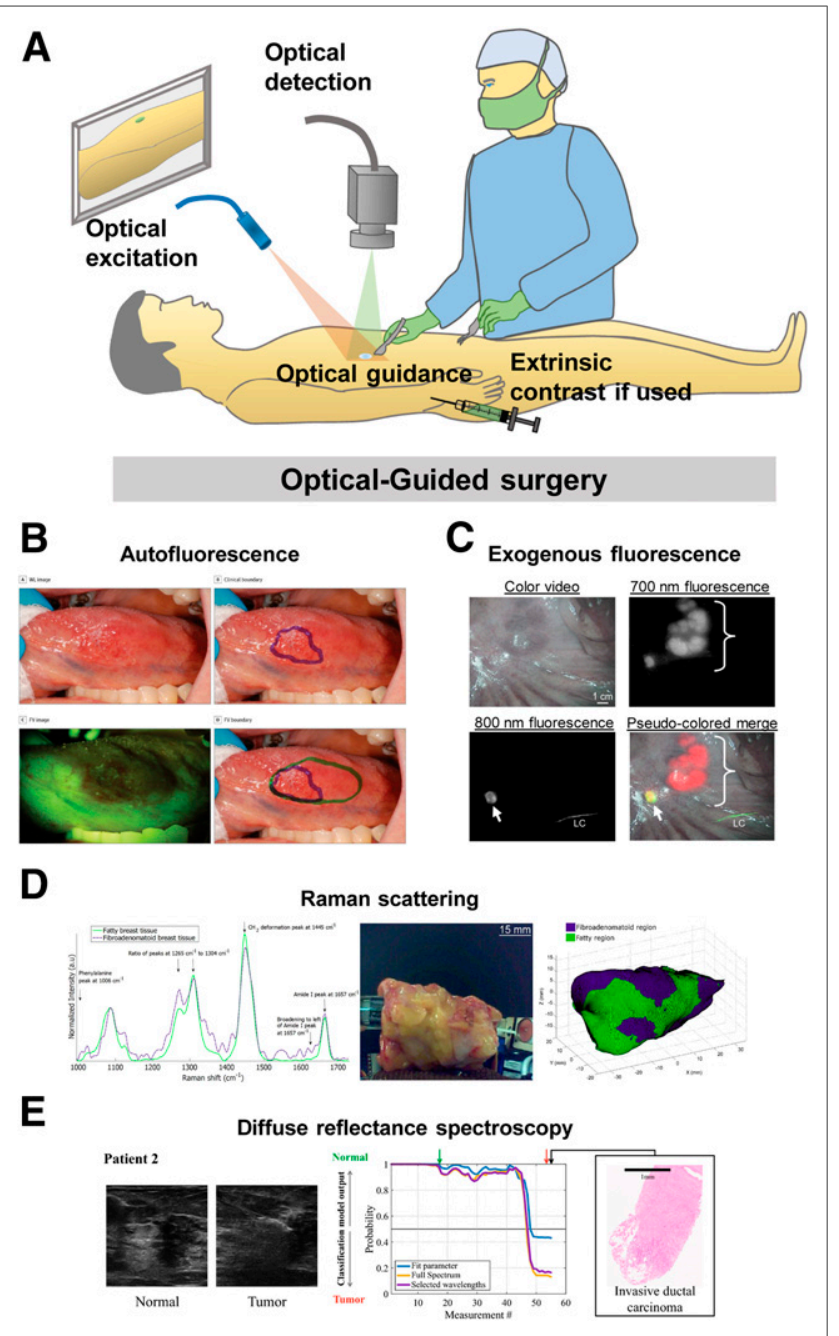

FIGURE 4. Optical guided surgery: schematic representation (A) and clinical examples of surgical guidance using autofluorescence imaging (48) (B), exogenous fluorescence imaging (22) (C), Raman scattering (36) (D), and diffuse reflectance spectroscopy (84) (E). a.u = arbitrary units; $\mathrm{FV}=$ fluorescence visualization; $\mathrm{WL}=$ white light. cancer in a pilot human study with high accuracy (47). Autofluorescence-guided surgical resection of oral cancer in a large singlecenter trial detected occult precancerous lesions that were missed by the operating surgeon and significantly reduced the locoregional rate of recurrence in patients with high-grade and earlystage oral cancer, compared with conventional surgery (Fig. 4) (48). Two-channel autofluorescence of dihydronicotinamide adenine dinucleotide (NADH) and flavin adenine dinucleotide (FAD) has also been used to accurately delineate a boundary between oral cancer or precancerous lesions and healthy oral mucosa using NADH/FAD and redox ratio (49). An emerging application of autofluorescence is in lung cancer, for which a differential NADH/FAD intensity ratio was observed in lung cancer versus normal tissue in preserved patient surgical samples (8). Autofluorescence video bronchoscopy has emerged as a new tool for lung cancer detection (50), has been shown to reveal a significantly larger tumor extent, and has influenced changes in treatment decisions in lung cancer patients when compared with conventional white-light video bronchoscopy (51). This technique was recently used to guide surgical resection in lung cancer and detected cancerous tissue with higher sensitivity than white-light video bronchoscopy, influencing changes in surgical decisions, and improving treatment outcomes $(52,53)$. Autofluorescence of frozen brain tumor biopsy samples allowed a combination of 3 molecular ratios to distinguish glioblastoma from healthy control tissue with high sensitivity and specificity, which were further improved through a combination of fluorescence lifetime, a measure of how long a fluorophore remains in the excited state before returning to the ground state after emitting a photon, with NADH/FAD and porphyrin/NADH autofluorescence (54).

Exogenous Fluorescence. Exogenous fluorescence imaging uses externally administered targeted fluorescent agents that accumulate in the anatomic ROI. The availability of a large selection of contrast agents (55) and devices (21) makes exogenous MIGS widely available to clinicians (Supplemental Table 5). Its clinical impact was first demonstrated using 5-aminolevulinic acid, which is a natural visible-range $(350-700 \mathrm{~nm})$ fluorophore that accumulates in glioblastomas $(56,57)$. 5-aminolevulinic acid fluorescence MIGS increased progression-free survival in glioblastoma patients (58) and has become the standard of care across European countries. Acriflavine and its principal component proflavine have been applied for oral cancer detection using high-resolution microendoscopy $(59,60)$. Combination of wide-field autofluorescence imaging with proflavine-enhanced high-resolution microendoscopy has enabled investigators to scan wide areas for suggestive lesions, and then further examine the nuclear-to-cytoplasmic ratio within a small area using high-resolution microendoscopy for added specificity in detecting oral neoplasia (61-63). Folate receptor- $\alpha$ conjugated fluorescein isothiocyanate was used to target epithelial ovarian cancers in human patients, and targeted fluorescence in ovarian and breast cancer tissue was observed intraoperatively $(64,65)$ and enabled the identification of ovarian cancer lesions not detectable by the naked eye (65). It has also been successfully used for lung cancer identification intraoperatively (66). Fluorescein has been used to guide resection of high-grade gliomas, as it was found to accumulate in glioma tissue (67). Visible-dye conjugates for MIGS are advantageous because they are readily accessible and clinically translatable because of previous use in human patients, and the shallow penetration of light in this region allows visualization of lesions closer to the tissue surface without interference from uninvolved deep fluorescent tissue. However, 
the visible-wavelength fluorophores did create false-positive signals in both breast and ovarian cancer patients, caused by background tissue autofluorescence (65). To minimize confounding autofluorescence during MIGS, most researchers are now using near-infrared (NIR) fluorescent dyes. Imaging in this wavelength region has the additional advantage of allowing the interrogation of deeper tissue than is possible with visible dyes. Indocyanine green is a Food and Drug Administration-approved NIR fluorescent contrast agent that has been widely used for MIGS (55), especially for highly sensitive detection of SLNs comparable to or better than standard-of-care radioactive and blue dye tracking (Fig. 4) (22,28,29). A NIR folate receptor-targeted fluorophore, OTL38, was recently tested in ovarian cancer patients and enabled an additional $29 \%$ of malignant lesions to be removed that were not identified by standard surgical practice (68). OTL38 also allowed highly sensitive intraoperative detection of malignant pulmonary nodules with very high sensitivity, including identification of nodules missed preoperatively (69). Development of multiple antibody-conjugated NIR dyes is under way, including epidermal growth factor receptor-targeting cetuximab-IRDye800, which was safely visualized in head and neck cancer (70), and vascular endothelial growth factor-A targeting bevacizumab-IRDye $800 \mathrm{CW}$, which was safely visualized in breast cancer patients (71). Recently, ABY-029, an epidermal growth factor receptor-targeted Affibody (Affibody AB) conjugated to IRDye800, was developed for MIGS in humans (18) to take advantage of the smaller size, higher affinity, and easier tissue clearance of Affibody molecules than of antibodies, enabling microdosing for in vivo image guidance. It is currently under investigation for first-in-human microdose studies in head and neck cancer, brain cancer, and sarcoma, with initial reports indicating a good correlation between ABY029 fluorescence and epidermal growth factor receptor expression (72). Additional tumor-targeted NIR fluorescent agents that are currently undergoing clinical evaluation are expected to receive regulatory approval soon (73) and may improve the outcomes of oncologic surgery in centers that do not have easy access to specialist equipment, personnel, and radiotracers.

\section{Raman Scattering (16)}

Raman scattering describes energy transfer between a photon and a vibrational mode in a molecule that results in a characteristic loss or gain of energy depending on the molecular bond and vibrational mode type. Plotting the energy difference between the incident light on the sample and the Raman-scattered light creates a Raman spectrum, sometimes referred to as a fingerprint, with varying intensities across the spectrum based on molecular content. These information-rich spectra can be used to probe the endogenous molecular content of healthy and cancerous tissue, as well as exogenous contrast agents, by comparing features such as intensity, peak width, and peak symmetry across different regions of the spectrum. Raman spectroscopy has been successfully used to determine tissue pathology, identifying subtle changes in tissue composition in freshly excised brain tissue (74). Further application in pediatric brain cancers also demonstrated strong agreement between Raman and conventional pathology (75). The diverse sources of contrast for Raman spectroscopy allow the use of tissue-specific properties to determine which contrast source is most valuable. For example, emphasis on specific tissue Raman signatures has enabled identification of ex vivo oral cancers $(76,77)$, assessment of breast cancer margins from in vivo (9) and ex vivo specimens $(78,79)$, identification of soft-tissue sarcomas $(80)$, and characterization of the prostate using specific Raman signatures during robotic prostate surgery (Fig. 4) (36). Recently, extrinsic surface-enhanced Raman-scattering nanoparticles targeting human epidermal growth factor receptor 2, estrogen receptor, epidermal growth factor receptor, and CD44 antigen were successfully used for intraoperative margin assessment of excised breast tumors with high accuracy in under 15 min (81). Endogenous Raman imaging can take advantage of the intrinsic molecular contrast between healthy and cancerous tissue without the added barriers of translating exogenous agents; however, the intrinsic signal from soft tissue is very weak and requires expensive, sophisticated hardware for operation and long scanning times. Pairing Raman imaging with contrast agents allows the use of much simpler hardware while maintaining multiplexing capabilities to track multiple targets quickly. Raman-guided surgery is well poised for wide clinical adoption for intraoperative tumor margin assessment (Supplemental Table 5).

\section{Diffuse Reflectance Spectroscopy}

Diffuse reflectance spectroscopy uses specific wavelengths or a range of wavelengths to illuminate intrinsic biomolecules and applies the spectral information in the reflected light to obtain the absorption and scattering profile of the anatomic ROI. Because of altered tissue morphology in diseased tissue, reflectance spectroscopy can identify the anatomic ROI (Supplemental Table 5). Diffuse reflectance spectroscopy has been used for assessing breast cancer margins intraoperatively (Fig. 4) (82-84) and for guiding colorectal cancer surgery (85). Hyperspectral imaging collects wide-field images at a range of wavelengths to estimate tissue properties and distinguish the anatomic ROI (10); it has been used for accurate identification of head and neck cancers in freshly excised specimens (86), intraoperative margin assessment in breast cancer (87), intraoperative detection of brain cancer (88), and guidance during various gastrointestinal surgeries (89).

\section{MULTIMODAL IMAGING}

A simple MIGS system that is capable of localizing cancer preoperatively and aiding intraoperative identification and resection in real time at both deep and superficial depth is not currently available. For this reason, several researchers are developing multimodal imaging platforms that combine the strengths of two or more MI modalities to overcome the limitations of individual modalities and improve the accuracy of surgical guidance (Fig. 5). In this context, multimodal imaging involves the use of more than one imaging method to acquire diverse data for integrated image analysis and information retrieval. A few such systems and methods are in different stages of clinical trials for MIGS (Supplemental Table 6).

\section{Cerenkov Luminescence Imaging}

With the advent of highly sensitive imaging systems, it has become possible to capture the low-radiance Cerenkov luminescence photons from PET radionuclides (Fig. 5). The exciting opportunity to integrate noninvasive preoperative nuclear imaging with intraoperative localization of tumors using $\gamma$ - or optical sensors could shorten the operating time, minimize patient discomfort, and improve surgical outcomes (Supplemental Table 6). PET radionuclides emit a broad spectrum of light with high intensity in the ultraviolet region that decreases at a rate of $1 / \lambda^{2}$ into the visible and NIR wavelengths $(300-900 \mathrm{~nm})$. The effect is more noticeable in a dielectric medium, where a charged particle travels 


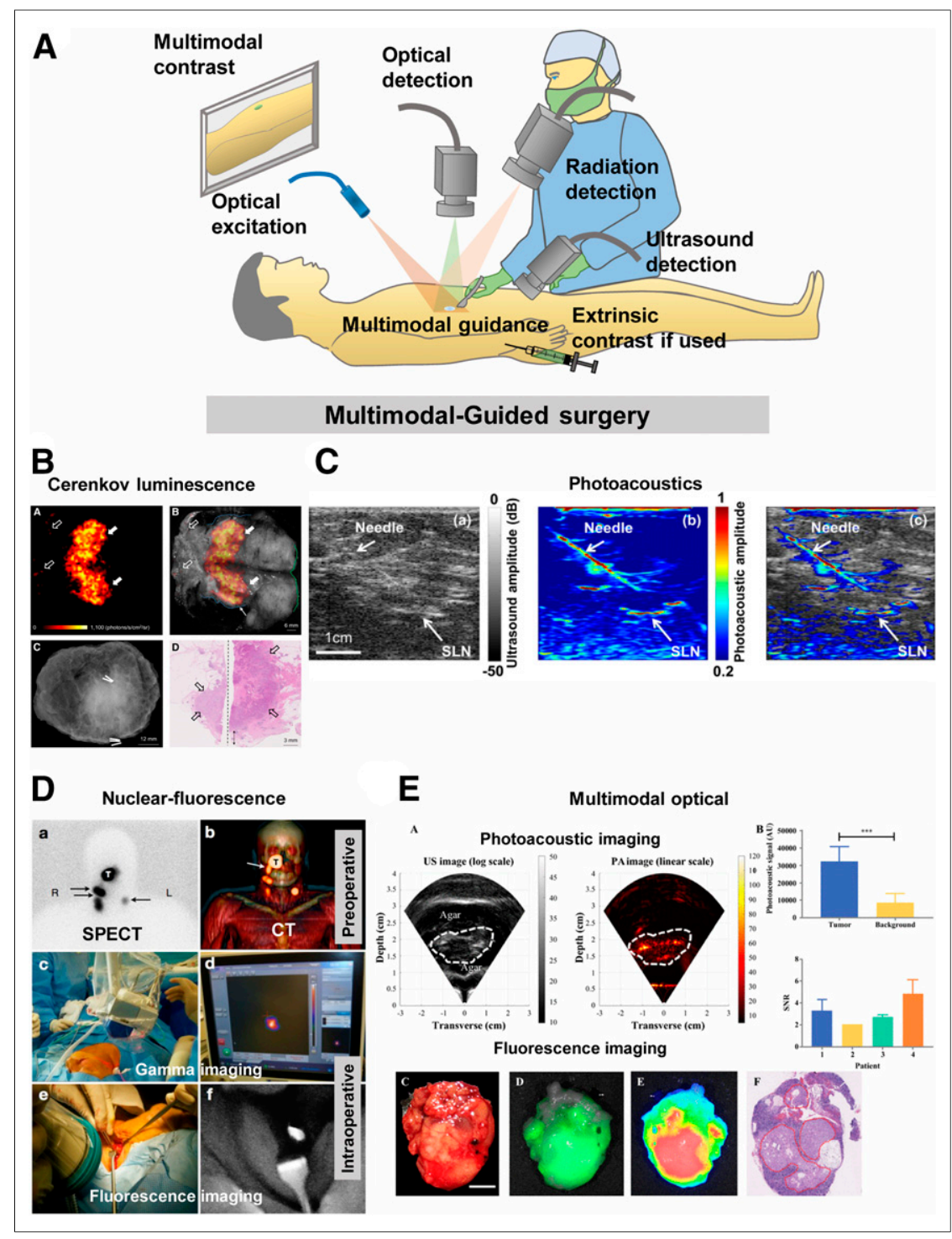

FIGURE 5. Multimodal guided surgery: schematic representation (A) and clinical examples of surgical guidance using Cerenkov luminescence imaging (91) (B), photoacoustic imaging (97) (C), nuclear-fluorescence imaging (105) (D), and multimodal optical imaging (112) (E). AU = arbitrary units; PA = photoacoustic; SNR = signal-to-noise ratio; US = ultrasound.

faster than the speed of light (90). Most PET radiotracers can generate Cerenkov luminescence with a maximum emission intensity at $350 \mathrm{~nm}$, thus allowing only superficial imaging. Additionally, Cerenkov photons are about 1,000 times weaker than commonly used fluorophores and can be imaged only by using long integration times with surgical lights turned off. Cerenkov luminescence imaging does not need an excitation light source, thereby enhancing the detection of the weak light from deep tissue similar to bioluminescence imaging. Cerenkov luminescence imaging has enabled tumor resection assessment in breast cancer patients (91) and endoscopic detection of gastrointestinal cancers (92). By leveraging the light from radionuclides used in the standard of care, Cerenkov luminescence imaging does not add to the cost of radiopharmaceuticals. It is conceivable that the same system might be used for both preoperative and intraoperative imaging, particularly for shallow tumors. The high spatial resolution of the optical cameras could further report the extent of cancer heterogeneity, allowing the surgeon to provide additional information to a pathologist on special features of the lesion. Despite these advantages, the optical imaging sensors are optimized in the visible and NIR regions of light, where the photon counts for this technique are low. For an intraoperative device, it may be valuable to harvest the largely unused photons in the ultraviolet region via down-conversion of nanoparticles for improved Cerenkov luminescence imaging.

\section{Photoacoustics}

Photoacoustic imaging uses a shortpulsed laser to excite intrinsic or extrinsic absorbers, which can undergo thermoelastic expansion after optical absorption and generate a pressure wave detectable by an ultrasound transducer (Fig. 5). It combines the high resolution of optical imaging with the depth penetration of ultrasound $(93,94)$. This technology has been used in the clinic for breast tumor margin assessment (9396), SLN mapping and assessment of the metastatic status in breast cancer (97), melanoma resection (98), and neurovascular bundle identification during prostate surgery (Supplemental Table 6) (99). Photoacoustic microscopy has also been used for label-free evaluation of breast tumor margins, with images that were comparable to processed histology slides (96). Recent studies have extended intraoperative photoacoustic imaging to colon and ovarian cancers, yielding a wealth of information for determining whether a tumor is benign or malignant based on tumor-associated vascularity and hypoxia $(100,101)$. The seamless interplay between contrast provided by endogenous and exogenous contrast sources further enhances the quality and content of information derived from this method. Furthermore, the rapid image-processing features of new systems have boosted real-time image acquisition and display, which will accelerate clinical decisions in the OR. As newer handheld and cheaper systems continue to emerge, the full potential of this technology for imageguided surgery will be achieved. Still, the technology is more expensive than simple fluorescence MIGS systems. In addition, transmission of sound requires direct contact of the probe with tissue, a condition that may not be feasible in some surgical situations.

\section{Nuclear Fluorescence}

Nuclear and fluorescence imaging methods have complementary properties to capture quantitative depth-independent nuclear information with high-resolution, real-time fluorescence images (102). This approach enables both diagnostic imaging for surgical planning and intraoperative surgical guidance (Fig. 5). A variety of methods are available to achieve a complementary effect. An obvious case is to combine the exogenous radiotracers with 
endogenous tissue autofluorescence for presurgical or deep-tissue imaging and real-time tumor boundary assessment, respectively, in the OR. Alternatively, a radiotracer can be mixed with a fluorescent molecule that targets the same tissue to achieve depthindependent cancer localization and real-time, high-resolution fluorescence-guided tumor resection. If both imaging agents are approved for clinical use, the path to clinical translation is less challenging than when new contrast agents are used (Supplemental Table 6). This method was first demonstrated using indocyanine green- ${ }^{99 \mathrm{~m}} \mathrm{Tc}$-nancolloid for preoperative SPECT/CT and intraoperative $\gamma$ - and NIR fluorescence imaging during laparoscopic SLN mapping in prostate $(103,104)$, head and neck $(104,105)$, melanoma $(104,106)$, penile $(104)$, and vulvar cancer (104) surgeries. It consistently detected more SLNs than blue dye tracking alone for a variety of cancers (104). Although this method is effective for SNL identification and biopsy, the disparate biodistribution profiles of each agent may decrease the accuracy of colocalizing the images for tumor resection. A widely used approach to overcome this disparity is conjugation of the radionuclide and the fluorescent dye to a tumor-targeting carrier. Recently, ${ }^{111}$ In-DOTA-girentuximab-IRDye800CW was successfully used for preoperative SPECT/CT-based tumor localization and intraoperative $\gamma$-fluorescence imaging for tumor margin assessment of clear cell renal cell carcinoma (107). ${ }^{68} \mathrm{Ga}$-IRDye800CW-BBN has also allowed accurate glioblastoma detection and showed an excellent correlation between preoperative PET and intraoperative fluorescence signal localization (108). Multimodal imaging has also been adapted for robotic surgery using an innovative dropin laparoscopic $\gamma$-detector that improved SLN detection sensitivity in prostate cancer patients (6). The future of this MIGS strategy remains bright as more handheld or wearable devices capable of capturing both nuclear and optical signals become available, providing a single system for acquiring presurgical, intraoperative, and postsurgical images of anatomic ROIs.

\section{Multimodal Optical}

Multimodal optical imaging combines optical modalities for accurate disease detection intraoperatively (Fig. 5; Supplemental Table 6). Reflectance spectroscopy imaging was coupled with fluorescence polarization imaging for accurate delineation of basal cell carcinoma (109). Multimodal polarization, reflectance, and fluorescence imaging methods were also used for intraoperative detection of breast cancer (110). Trimodal optical imaging provided identification of oral cancer (111) using autofluorescence spectroscopy, diffuse reflectance spectroscopy, and light-scattering spectroscopy. A combination of exogenous fluorescence and photoacoustic imaging has been used for intraoperative detection of pancreatic cancer (112). Multimodal optical approaches have a high potential impact on intraoperative surgical guidance based on simpler hardware, high resolution, and their ability to make use of many different sources of contrast depending on the optical modality and type of contrast used. We expect multimodal optical approaches to continue impacting this field and enable multiplexed surgical guidance, including identification of tumors, vasculature, nerves, and other important anatomy simultaneously.

\section{CONCLUSION}

MIGS is already improving surgical outcomes through nuclear imaging modalities that have been the standard of care for several subspecialties. As optical imaging matures further, more optical modalities will be available to clinicians for use in their practice and research, fueling the next wave of surgical innovation. Adoption of multimodal approaches may be expected to increase because of multidimensional information streams and potential combination with machine-learning algorithms to gain new insights to deliver better patient care. MIGS surgery will continue to play a critical role as the state of the art in surgical practice evolves in the future.

\section{DISCLOSURE}

Suman Mondal, Christine O'Brien, Kevin Bishop, and Samuel Achilefu were supported in part by the National Institutes of Health grants (R01 CA171651, U54 CA199092, R01 EB021048, P50 CA094056, P30 CA091842, S10 OD020129, S10 OD016237, and S10 RR031625), the Department of Defense Breast Cancer Research Program (W81XWH-16-1-0286), and the Alvin J. Siteman Cancer Center Investment Program Research Development Award. No other potential conflict of interest relevant to this article was reported.

\section{REFERENCES}

1. Tempany CMC, Jayender J, Kapur T, et al. Multimodal imaging for improved diagnosis and treatment of cancers. Cancer. 2015;121:817-827.

2. Mondal SB, Gao S, Zhu N, Liang R, Gruev V, Achilefu S. Real-time fluorescence image-guided oncologic surgery. Adv Cancer Res. 2014;124:171-211.

3. Stammes MA, Bugby SL, Porta T, et al. Modalities for image- and molecularguided cancer surgery. Br J Surg. 2018;105:e69-e83.

4. Alam IS, Steinberg I, Vermesh O, et al. Emerging intraoperative imaging modalities to improve surgical precision. Mol Imaging Biol. 2018;20:705-715.

5. Jolesz FA. Introduction. In: Jolesz FA, ed. Intraoperative Imaging and ImageGuided Therapy. New York, NY: Springer New York; 2014:1-23.

6. Meershoek P, van Oosterom MN, Simon H, et al. Robot-assisted laparoscopic surgery using DROP-IN radioguidance: first-in-human translation. Eur J Nucl Med Mol Imaging. 2019;46:49-53.

7. Wu C, Gleysteen J, Teraphongphom NT, Li Y, Rosenthal E. In-vivo optical imaging in head and neck oncology: basic principles, clinical applications and future directions. Int J Oral Sci. 2018;10:10.

8. Wang M, Long F, Tang F, et al. Autofluorescence imaging and spectroscopy of human lung cancer. Appl Sci. 2017;7:32.

9. Haka AS, Volynskaya Z, Gardecki JA, et al. In vivo margin assessment during partial mastectomy breast surgery using Raman spectroscopy. Cancer Res. 2006;66:3317-3322.

10. Lu G, Fei B. Medical hyperspectral imaging: a review. J Biomed Opt. 2014;19: 10901.

11. Mattison SP, Kim W, Park J, Applegate BE. Molecular imaging in optical coherence tomography. Curr Mol Imaging. 2014;3:88-105.

12. James ML, Gambhir SS. A molecular imaging primer: modalities, imaging agents, and applications. Physiol Rev. 2012;92:897-965.

13. Bunschoten A, van den Berg NS, Olmos RAV, Blokland JA, van Leeuwen FW. Tracers applied in radioguided surgery. In: Herrmann K, Nieweg OE, Povoski SP, eds. Radioguided Surgery. Cham, Switzerland: Springer International Publishing; 2016:75-101.

14. Maurer T, Graefen M, van der Poel H, et al. Prostate-specific membrane antigenguided surgery. J Nucl Med. 2020;61:6-12.

15. Achilefu S, Dorshow RB, Bugaj JE, Rajagopalan R. Novel receptor-targeted fluorescent contrast agents for in vivo tumor imaging. Invest Radiol. 2000;35: $479-485$.

16. Shen $\mathrm{D}, \mathrm{Xu} \mathrm{B}$, Liang K, et al. Selective imaging of solid tumours via the calcium-dependent high-affinity binding of a cyclic octapeptide to phosphorylated Annexin A2. Nat Biomed Eng. 2020;4:298-313.

17. Tummers WS, Kimura RH, Abou-Elkacem L, et al. Development and preclinical validation of a cysteine knottin peptide targeting integrin $\alpha \mathrm{v} \beta 6$ for near-infrared fluorescent-guided surgery in pancreatic cancer. Clin Cancer Res. 2018;24:16671676.

18. Samkoe KS, Gunn JR, Marra K, et al. Toxicity and pharmacokinetic profile for single-dose injection of ABY-029: a fluorescent anti-EGFR synthetic Affibody molecule for human use. Mol Imaging Biol. 2017;19:512-521. 
19. Miller SE, Tummers WS, Teraphongphom N, et al. First-in-human intraoperative near-infrared fluorescence imaging of glioblastoma using cetuximabIRDye800. J Neurooncol. 2018;139:135-143.

20. $\mathrm{Ng} \mathrm{AH}$, Blackshaw PE, Alqahtani MS, et al. A novel compact small field of view hybrid gamma camera: first clinical results. Nucl Med Commun. 2017;38: 729-736.

21. DSouza AV, Lin H, Henderson ER, Samkoe KS, Pogue BW. Review of fluorescence guided surgery systems: identification of key performance capabilities beyond indocyanine green imaging. J Biomed Opt. 2016;21:80901.

22. Troyan SL, Kianzad V, Gibbs-Strauss SL, et al. The FLARE intraoperative near-infrared fluorescence imaging system: a first-in-human clinical trial in breast cancer sentinel lymph node mapping. Ann Surg Oncol. 2009;16:29432952.

23. Tobis S, Knopf JK, Silvers CR, et al. Near infrared fluorescence imaging after intravenous indocyanine green: initial clinical experience with open partial nephrectomy for renal cortical tumors. Urology. 2012;79:958-964

24. Thomas G, McWade MA, Nguyen JQ, et al. Innovative surgical guidance for label-free real-time parathyroid identification. Surgery. 2019;165:114-123.

25. Lees JE, Bugby SL, Alqahtani MS, et al. A multimodality hybrid gammaoptical camera for intraoperative imaging. Sensors (Basel). 2017;17:E554.

26. Gotoh K, Yamada T, Ishikawa $\mathrm{O}$, et al. A novel image-guided surgery of hepatocellular carcinoma by indocyanine green fluorescence imaging navigation. J Surg Oncol. 2009;100:75-79.

27. Mieog JS, Vahrmeijer AL, Hutteman M, et al. Novel intraoperative near-infrared fluorescence camera system for optical image-guided cancer surgery. Mol Imaging. 2010;9:223-231.

28. Mondal SB, Gao S, Zhu N, et al. Optical see-through cancer vision goggles enable direct patient visualization and real-time fluorescence-guided oncologic surgery. Ann Surg Oncol. 2017;24:1897-1903.

29. Mondal SB, Gao S, Zhu N, et al. Binocular goggle augmented imaging and navigation system provides real-time fluorescence image guidance for tumor resection and sentinel lymph node mapping. Sci Rep. 2015;5:12117.

30. Lee JYK, Cho SS, Zeh R, et al. Folate receptor overexpression can be visualized in real time during pituitary adenoma endoscopic transsphenoidal surgery with near-infrared imaging. J Neurosurg. 2018;129:390-403.

31. Maitland KC, Gillenwater AM, Williams MD, El-Naggar AK, Descour MR, Richards-Kortum RR. In vivo imaging of oral neoplasia using a miniaturized fiber optic confocal reflectance microscope. Oral Oncol. 2008;44:1059-1066.

32. Haxel BR, Goetz M, Kiesslich R, Gosepath J. Confocal endomicroscopy: a novel application for imaging of oral and oropharyngeal mucosa in human. Eur Arch Otorhinolaryngol. 2010;267:443-448.

33. Pavlov V, Meyronet D, Meyer-Bisch V, et al. Intraoperative probe-based confocal laser endomicroscopy in surgery and stereotactic biopsy of low-grade and highgrade gliomas: a feasibility study in humans. Neurosurgery. 2016;79:604-612.

34. Chen CK, Hsieh LC, Hsu TH. Novel three-dimensional image system for endoscopic ear surgery. Eur Arch Otorhinolaryngol. 2018;275:2933-2939.

35. Leonard S, Reiter A, Sinha A, Ishii M, Taylor RH, Hager GD. Image-based navigation for functional endoscopic sinus surgery using structure from motion. Paper presented at: SPIE Medical Imaging; March 21, 2016; San Diego, California.

36. Pinto M, Zorn KC, Tremblay JP, et al. Integration of a Raman spectroscopy system to a robotic-assisted surgical system for real-time tissue characterization during radical prostatectomy procedures. J Biomed Opt. 2019;24:1-10.

37. Daskalaki D, Aguilera F, Patton K, Giulianotti PC. Fluorescence in robotic surgery. J Surg Oncol. 2015;112:250-256.

38. Heller S, Zanzonico P. Nuclear probes and intraoperative gamma cameras. Semin Nucl Med. 2011;41:166-181.

39. Povoski SP, Neff RL, Mojzisik CM, et al. A comprehensive overview of radioguided surgery using gamma detection probe technology. World J Surg Oncol. 2009;7:11.

40. De Cicco C, Pizzamiglio M, Trifiro G, et al. Radioguided occult lesion localisation (ROLL) and surgical biopsy in breast cancer: technical aspects. $Q \mathrm{~J} \mathrm{Nucl}$ Med. 2002;46:145-151.

41. Sondak VK, King DW, Zager JS, et al. Combined analysis of phase III trials evaluating [ $\left.{ }^{99 \mathrm{~m}} \mathrm{Tc}\right]$ tilmanocept and vital blue dye for identification of sentinel lymph nodes in clinically node-negative cutaneous melanoma. Ann Surg Oncol. 2013;20:680-688.

42. Wallace AM, Han LK, Povoski SP, et al. Comparative evaluation of [ ${ }^{99 \mathrm{~m}} \mathrm{Tc}$ ] tilmanocept for sentinel lymph node mapping in breast cancer patients: results of two phase 3 trials. Ann Surg Oncol. 2013;20:2590-2599.

43. Gulec SA, Hoenie E, Hostetter R, Schwartzentruber D. PET probe-guided surgery: applications and clinical protocol. World J Surg Oncol. 2007;5:65.

44. Leong SP, Wu M, Lu Y, et al. Intraoperative imaging with a portable gamma camera may reduce the false-negative rate for melanoma sentinel lymph node surgery. Ann Surg Oncol. 2018;25:3326-3333.
45. Wendler T, Herrmann K, Schnelzer A, et al. First demonstration of 3-D lymphatic mapping in breast cancer using freehand SPECT. Eur J Nucl Med Mol Imaging. 2010;37:1452-1461.

46. Schilling C, Gnansegaran G, Thavaraj S, McGurk M. Intraoperative sentinel node imaging versus SPECT/CT in oral cancer: a blinded comparison. Eur J Surg Oncol. 2018;44:1901-1907.

47. Lane PM, Gilhuly T, Whitehead P, et al. Simple device for the direct visualization of oral-cavity tissue fluorescence. J Biomed Opt. 2006;11:024006.

48. Poh CF, Anderson DW, Durham JS, et al. Fluorescence visualization-guided surgery for early-stage oral cancer. JAMA Otolaryngol Head Neck Surg. 2016;142:209_ 216.

49. Huang T-T, Chen K-C, Wong T-Y, et al. Two-channel autofluorescence analysis for oral cancer. J Biomed Opt. 2018;24:1-10.

50. Nakajima T, Yasufuku K. Early lung cancer: methods for detection. In: DíazJimenez JP, Rodriguez AN, eds. Interventions in Pulmonary Medicine. Cham, Switzerland: Springer International Publishing; 2018:245-256.

51. Zaric B, Becker HD, Perin B, et al. Autofluorescence imaging videobronchoscopy improves assessment of tumor margins and affects therapeutic strategy in central lung cancer. Jpn J Clin Oncol. 2010;40:139-145.

52. Ye G, Hao W, Gening J, et al. The application of autofluorescence bronchoscopy in guiding surgical resection range for lung cancer [in Chinese]. Chung Hua Chieh Ho Ho Hu Hsi Tsa Chih. 2015;38:170-173.

53. Peng A, Li M, Zhang G, Wang C. The value of autofluorescence bronchoscopy in assessment of tumor extent and guide of therapeutic strategy in central lung cancer [in Chinese]. Chung Hua Nei Ko Tsa Chih.2015;54:40-43.

54. Poulon F, Chalumeau A, Jamme F, et al. Multimodal analysis of central nervous system tumor tissue endogenous fluorescence with multiscale excitation. Front Phys. 2018;6:109.

55. Nagaya T, Nakamura YA, Choyke PL, Kobayashi H. Fluorescence-guided surgery. Front Oncol. 2017;7:314.

56. Stummer W, Novotny A, Stepp H, Goetz C, Bise K, Reulen HJ. Fluorescenceguided resection of glioblastoma multiforme utilizing 5-ALA-induced porphyrins: a prospective study in 52 consecutive patients. J Neurosurg. 2000;93:1003.

57. Stummer W, Stepp H, Möller G, Ehrhardt A, Leonhard M, Reulen HJ. Technical principles for protoporphyrin-ix-fluorescence guided microsurgical resection of malignant glioma tissue. Acta Neurochir (Wien). 1998;140:995-1000.

58. Stummer W, Pichlmeier U, Meinel T, Wiestler OD, Zanella F, Reulen H-J. Fluorescence-guided surgery with 5 -aminolevulinic acid for resection of malignant glioma: a randomised controlled multicentre phase III trial. Lancet Oncol. 2006;7: 392-401.

59. Muldoon TJ, Pierce MC, Nida DL, Williams MD, Gillenwater A, RichardsKortum R. Subcellular-resolution molecular imaging within living tissue by fiber microendoscopy. Opt Express. 2007;15:16413-16423.

60. Muldoon TJ, Roblyer D, Williams MD, Stepanek VMT, Richards-Kortum R, Gillenwater AM. Noninvasive imaging of oral neoplasia with a high-resolution fiber-optic microendoscope. Head Neck. 2012;34:305-312.

61. Pierce MC, Schwarz RA, Bhattar VS, et al. Accuracy of in vivo multimodal optical imaging for detection of oral neoplasia. Cancer Prev Res (Phila). 2012;5: 801-809.

62. Quang T, Tran EQ, Schwarz RA, et al. Prospective evaluation of multimodal optical imaging with automated image analysis to detect oral neoplasia in vivo. Cancer Prev Res (Phila). 2017;10:563-570.

63. Yang EC, Vohra IS, Badaoui H, et al. Development of an integrated multimodal optical imaging system with real-time image analysis for the evaluation of oral premalignant lesions. J Biomed Opt. 2019;24:1-10.

64. van Dam GM, Themelis G, Crane LMA, et al. Intraoperative tumor-specific fluorescence imaging in ovarian cancer by folate receptor- $\alpha$ targeting: first inhuman results. Nat Med. 2011;17:1315.

65. Tummers QRJG, Hoogstins CES, Gaarenstroom KN, et al. Intraoperative imaging of folate receptor alpha positive ovarian and breast cancer using the tumor specific agent EC17. Oncotarget. 2016;7:32144-32155.

66. Okusanya OT, DeJesus EM, Jiang JX, et al. Intraoperative molecular imaging can identify lung adenocarcinomas during pulmonary resection. J Thorac Cardiovasc Surg. 2015;150:28-35.e1.

67. Acerbi F, Broggi M, Schebesch K-M, et al. Fluorescein-guided surgery for resection of high-grade gliomas: a multicentric prospective phase ii study (FLUOGLIO). Clin Cancer Res. 2018;24:52-61.

68. Hoogstins CES, Tummers QRJG, Gaarenstroom KN, et al. A novel tumor-specific agent for intraoperative near-infrared fluorescence imaging: a translational study in healthy volunteers and patients with ovarian cancer. Clin Cancer Res. 2016;22: 2929-2938.

69. Predina JD, Newton AD, Keating J, et al. Intraoperative molecular imaging combined with positron emission tomography improves surgical management of peripheral malignant pulmonary nodules. Ann Surg. 2017;266:479-488. 
70. Rosenthal EL, Warram JM, de Boer E, et al. Safety and tumor specificity of cetuximab-IRDye800 for surgical navigation in head and neck cancer. Clin Cancer Res. 2015;21:3658-3666.

71. Lamberts LE, Koch M, de Jong JS, et al. Tumor-specific uptake of fluorescent bevacizumab-IRDye $800 \mathrm{CW}$ microdosing in patients with primary breast cancer: a phase I feasibility study. Clin Cancer Res. 2017;23:2730-2741.

72. Samkoe KS, Sardar HS, Gunn J, et al. Measuring microdose ABY-029 fluorescence signal in a primary human soft-tissue sarcoma resection. Paper presented at: SPIE BiOS; May 22, 2019; San Francisco, California.

73. Joshi BP, Wang TD. Targeted optical imaging agents in cancer: focus on clinical applications. Contrast Media Mol Imaging. 2018;2018:2015237.

74. Orringer DA, Pandian B, Niknafs YS, et al. Rapid intraoperative histology of unprocessed surgical specimens via fibre-laser-based stimulated Raman scattering microscopy. Nat Biomed Eng. 2017;1:0027.

75. Hollon TC, Lewis S, Pandian B, et al. Rapid intraoperative diagnosis of pediatric brain tumors using stimulated Raman histology. Cancer Res. 2018;78:278-289.

76. Barroso EM, Smits RW, van Lanschot CG, et al. Water concentration analysis by Raman spectroscopy to determine the location of the tumor border in oral cancer surgery. Cancer Res. 2016;76:5945-5953.

77. Barroso EM, Smits RWH, Bakker Schut TC, et al. Discrimination between oral cancer and healthy tissue based on water content determined by Raman spectroscopy. Anal Chem. 2015;87:2419-2426.

78. Keller MD, Vargis E, Mahadevan-Jansen A, et al. Development of a spatially offset Raman spectroscopy probe for breast tumor surgical margin evaluation. J Biomed Opt. 2011;16:077006.

79. Thomas G, Nguyen TQ, Pence IJ, et al. Evaluating feasibility of an automated 3-dimensional scanner using Raman spectroscopy for intraoperative breast margin assessment. Sci Rep. 2017;7:13548.

80. Nguyen JQ, Gowani ZS, O'Connor M, et al. Intraoperative Raman spectroscopy of soft tissue sarcomas. Lasers Surg Med. 2016;48:774-781.

81. Wang YW, Reder NP, Kang S, et al. Raman-encoded molecular imaging with topically applied SERS nanoparticles for intraoperative guidance of lumpectomy. Cancer Res. 2017;77:4506-4516.

82. Kennedy S, Geradts J, Bydlon T, et al. Optical breast cancer margin assessment: an observational study of the effects of tissue heterogeneity on optical contrast. Breast Cancer Res. 2010;12:R91.

83. Bydlon TM, Kennedy SA, Richards LM, et al. Performance metrics of an optical spectral imaging system for intra-operative assessment of breast tumor margins. Opt Express. 2010;18:8058-8076.

84. de Boer LL, Bydlon TM, van Duijnhoven F, et al. Towards the use of diffuse reflectance spectroscopy for real-time in vivo detection of breast cancer during surgery. J Transl Med. 2018;16:367.

85. Baltussen EJM, Snæbjörnsson P, de Koning SGB, et al. Diffuse reflectance spectroscopy as a tool for real-time tissue assessment during colorectal cancer surgery. J Biomed Opt. 2017;22:1-6.

86. Lu G, Little JV, Wang X, et al. Detection of head and neck cancer in surgical specimens using quantitative hyperspectral imaging. Clin Cancer Res. 2017;23: 5426-5436.

87. Kho E, de Boer LL, Van de Vijver KK, et al. Hyperspectral imaging for resection margin assessment during cancer surgery. Clin Cancer Res. 2019;25:35723580 .

88. Fabelo H, Ortega S, Ravi D, et al. Spatio-spectral classification of hyperspectral images for brain cancer detection during surgical operations. PLoS One. 2018;13: e0193721.

89. Ortega S, Fabelo H, Iakovidis DK, Koulaouzidis A, Callico GM. Use of hyperspectral/multispectral imaging in gastroenterology: shedding some different light into the dark. J Clin Med. 2019;8:36.

90. Grootendorst MR, Cariati M, Kothari A, Tuch D, Purushotham A. Cerenkov luminescence imaging (CLI) for image-guided cancer surgery. Clin Transl Imaging. 2016;4:353-366.

91. Grootendorst MR, Cariati M, Pinder SE, et al. Intraoperative assessment of tumor resection margins in breast-conserving surgery using ${ }^{18} \mathrm{~F}$-FDG Cerenkov luminescence imaging: a first-in-human feasibility study. J Nucl Med. 2017;58: 891-898.

92. Hu H, Cao X, Kang F, et al. Feasibility study of novel endoscopic Cerenkov luminescence imaging system in detecting and quantifying gastrointestinal disease: first human results. Eur Radiol. 2015;25:1814-1822.

93. Xiao G, Bonmati E, Thompson S, et al. Electromagnetic tracking in image-guided laparoscopic surgery: comparison with optical tracking and feasibility study of a combined laparoscope and laparoscopic ultrasound system. Med Phys. 2018;45:5094-5104.

94. Goh Y, Balasundaram G, Moothanchery M, et al. Multispectral optoacoustic tomography in assessment of breast tumor margins during breast-conserving surgery: a first-in-human case study. Clin Breast Cancer. 2018;18:e1247-e1250.

95. Kosik I, Brackstone M, Kornecki A, et al. Intraoperative photoacoustic screening of breast cancer: a new perspective on malignancy visualization and surgical guidance. J Biomed Opt. 2019;24:1-12.

96. Wong TTW, Zhang R, Hai P, et al. Fast label-free multilayered histology-like imaging of human breast cancer by photoacoustic microscopy. Sci Adv. 2017;3:e1602168.

97. Garcia-Uribe A, Erpelding TN, Krumholz A, et al. Dual-modality photoacoustic and ultrasound imaging system for noninvasive sentinel lymph node detection in patients with breast cancer. Sci Rep. 2015;5:15748.

98. Stoffels I, Morscher S, Helfrich I, et al. Metastatic status of sentinel lymph nodes in melanoma determined noninvasively with multispectral optoacoustic imaging. Sci Transl Med. 2015;7:317ra199.

99. Horiguchi A, Tsujita K, Irisawa K, et al. A pilot study of photoacoustic imaging system for improved real-time visualization of neurovascular bundle during radical prostatectomy. Prostate. 2016;76:307-315.

100. Yang G, Amidi E, Chapman WC, et al. Co-registered photoacoustic and ultrasound imaging of human colorectal cancer. J Biomed Opt. 2019;24:1-13.

101. Nandy S, Mostafa A, Hagemann IS, et al. Evaluation of ovarian cancer: initial application of coregistered photoacoustic tomography and US. Radiology. 2018;289:740-747.

102. Bugby SL, Lees JE, Perkins AC. Hybrid intraoperative imaging techniques in radioguided surgery: present clinical applications and future outlook. Clin Transl Imaging. 2017;5:323-341.

103. van der Poel HG, Buckle T, Brouwer OR, Valdés Olmos RA, van Leeuwen FWB. Intraoperative laparoscopic fluorescence guidance to the sentinel lymph node in prostate cancer patients: clinical proof of concept of an integrated functional imaging approach using a multimodal tracer. Eur Urol. 2011;60:826-833.

104. KleinJan GH, van Werkhoven E, van den Berg NS, et al. The best of both worlds: a hybrid approach for optimal pre- and intraoperative identification of sentinel lymph nodes. Eur J Nucl Med Mol Imaging. 2018;45:1915-1925.

105. Brouwer OR, Klop WMC, Buckle T, et al. Feasibility of sentinel node biopsy in head and neck melanoma using a hybrid radioactive and fluorescent tracer. Ann Surg Oncol. 2012;19:1988-1994.

106. KleinJan GH, Karakullukçu B, Klop WMC, Engelen T, van den Berg NS, van Leeuwen FWB Introducing navigation during melanoma-related sentinel lymph node procedures in the head-and-neck region. EJNMMI Res. 2017;7:65.

107. Hekman MC, Rijpkema M, Muselaers CH, et al. Tumor-targeted dual-modality imaging to improve intraoperative visualization of clear cell renal cell carcinoma: a first in man study. Theranostics. 2018;8:2161-2170.

108. Li D, Zhang J, Chi C, et al. First-in-human study of PET and optical dualmodality image-guided surgery in glioblastoma using ${ }^{68} \mathrm{Ga}$-IRDye $800 \mathrm{CW}$ BBN. Theranostics. 2018;8:2508-2520.

109. Salomatina E, Muzikansky A, Neel V, Yaroslavsky AN. Multimodal optical imaging and spectroscopy for the intraoperative mapping of nonmelanoma skin cancer. J Appl Phys. 2009;105:102010.

110. Patel R, Khan A, Wirth DJ, et al. Multimodal optical imaging for detecting breast cancer. J Biomed Opt. 2012;17:066008.

111. Müller MG, Valdez TA, Georgakoudi I, et al. Spectroscopic detection and evaluation of morphologic and biochemical changes in early human oral carcinoma. Cancer. 2003;97:1681-1692.

112. Tummers WS, Miller SE, Teraphongphom NT, et al. Intraoperative pancreatic cancer detection using tumor-specific multimodality molecular imaging. Ann Surg Oncol. 2018;25:1880-1888. 